\title{
Post-Psychotic Depression: An Updated Review of the Term and Clinical Implications
}

\author{
Margarita Guerrero-Jiménez ${ }^{\mathrm{a}}$ Carmen Maura Carrillo de Albornoz Calahorro ${ }^{a}$ \\ Braulio Girela-Serrano $^{b}$ Isabel Bodoano Sánchez ${ }^{a}$ Luis Gutiérrez-Rojas ${ }^{c, d}$ \\ aPsychiatry Service, Hospital Virgen de las Nieves Hospital, Granada, Spain; ${ }^{b}$ Division of Psychiatry, Department of \\ Brain Sciences, Imperial College, London, UK; CPsychiatry and Neurosciences Research Group (CTS-549), Institute of

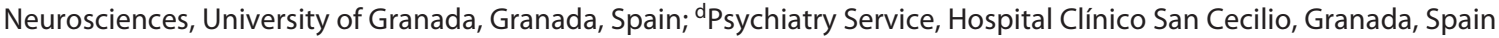

\section{Keywords}

Depressive disorders · Diagnosis · Insight · Schizophrenia · Suicide

\begin{abstract}
Background: Post-psychotic depression (PPD) is an important and frequent clinical phenomenon featuring controversial complexity in its nosological and aetiopathogenic cataloguing. Objectives: The main objective of this research was to review the published literature on PPD. The second objective was to indicate its clinical importance, either comorbid or as an entity of its own. To answer these questions, a historical review of the term is made and a search about the clinical, evolutionary, predisposal, and prognostic variables that characterize the PPD. Methods: The international recommendations were followed according to the Preferred Items for Reporting of Systematic Reviews and Meta-Analyses (PRISMA). The databases used were Web of Science and PubMed, with the deadline for the inclusion of articles in November 2019. The MeSH used were the following: "post" AND "psych *" AND "psich" AND "depr." Results: The search resulted in 64 articles. Only 19 of these met the pre-specified inclusion criteria and were finally included in the review. One article found that reading this literature was added due to its relevance. Despite its high prevalence (around 30\%), there is little research about the term PPD. Nevertheless, results
\end{abstract}

show data to improve the description of the syndrome, revealing differential characteristics from other depressive symptoms in chronic psychosis due to its clinical implications. Conclusions: Coinciding with the latest classification manuals that do not include the term, there appears to be an abandonment of its use despite its high prevalence. Data suggest that PPD is a nosological entity different from a secondary effect to antipsychotics, the negative symptoms of psychosis, and other clinical disorders that combine psychotic and depressive symptoms such as bipolar disorder, schizoaffective disorder, or depression with psychotic symptoms. PPD also has differential characteristics concerning further depressive symptoms, especially important clinical implications such as higher suicide risk and poorer quality of life.

(c) 2022 S. Karger AG, Basel

\section{Introduction}

Post-psychotic depression (PPD) is an important and frequent clinical phenomenon. Nevertheless, there is a controversial complexity in its nosological and aetiopathogenic cataloguing. There are many difficulties when it comes to specifying and agreeing on its exact prevalence, prognostic implications, and aetiology $[1,2]$. The following is because there are clinical limits that are dif-
Karger@karger.com www.karger.com/psp

Karger
(C) 2022 S. Karger AG, Basel

(2022 S. Karger AG, Basel
Correspondence to:

Luis Gutiérrez-Rojas, gutierrezrojasl@hotmail.com 
Table 1. Historical evolution of the term PPD

\begin{tabular}{ll}
\hline Author & Historical evolution of the term PPD \\
\hline Mayer-Gross [4] & "Future rejection and hopelessness as a way of reacting after a psychotic experience" \\
Bleuler [3] & $\begin{array}{l}\text { Depressive symptoms as a nuclear phenomenon in schizophrenia } \\
\text { Eissler [5] }\end{array}$ \\
"A relative clinical mutism phase" after the acute phase of schizophrenia \\
Conrad [9] & Separation between affective and non-affective psychosis \\
Semrad [6] & Even in the prepsychotic period, depressive symptoms were described and were called "delusional mood" \\
Mino and Ushijima [7] & $\begin{array}{l}\text { Narcissistic regression with an important use of denial, projection, and distortion (depressive functioning) } \\
\text { "Post-psychotic collapse": state of under-activity: loss of energy and vitality after an improvement of psychotic } \\
\text { symptoms }\end{array}$ \\
\hline
\end{tabular}

ficult to define, given that it is a heterogeneous entity with different diagnostic subtypes, having been catalogued with terms such as experiential reaction or adaptive disorder with depressed mood, secondary to the iatrogenicity of antipsychotics, such as "akinetic depression," "aphanisis," or "comorbid depressive disorder." In short, it has been used interchangeably to refer to any depressive symptoms that appear throughout the life of a patient with psychosis, without taking into account the aetiology of the process and creating great confusion [1].

It was Bleuler who argued for the first time that affective problems were part of the core of schizophrenia, while the symptoms we had always focused on, such as hallucinations and delusions, were merely accessory and common to other psychotic disorders [3]. However, another author, Mayer-Gross in 1920, had already referred to them when describing "future rejection and despair as a way of reacting after a psychotic experience" [4]. A few years later, Eissler (1951) presented a description of the syndrome as "a phase of relative clinical mutism" after the acute phase of schizophrenia [5]. In 1966, Semrad [6] proposed a dynamic explanation about the implications of the post-psychotic period, in which they suggested that at this stage, the patient progresses out of his position of narcissistic regression with an important use of denial, projection, and distortion functioning more like a depressed patient. A more recent description calls PPD the "post-psychotic collapse," a state of under-activity with a loss of energy and vitality after an improvement of psychotic symptoms [7].

Only a few years later, this vision gave way to the familiar distinction between "affective psychoses" and "non-affective psychoses" [8]. Back then, Conrad described in his "Incipient Schizophrenia" that even in the prepsychotic period, depressive symptoms can be described, which he called "delusional mood" [9]. Could it be that these symptoms are the beginning of this affective symptomatology that continues to manifest itself during the psychotic and post-psychotic stages (Table 1)?

In the last 2 decades, experts in the field have begun to relocate the importance of the affective plane as part of the disorder and the recovery processes of patients with psychosis. The affective plane of psychosis has begun to be treated under the name of "comorbidity," referring to "depression," "feelings of hopelessness," "suicidal ideation," "social anxiety," and "post-traumatic symptoms." In this period of years, some authors have given continuity to Bleuler's statement and have pointed out the "emotional dysfunction" as a nuclear aspect that evolves with the same sensitivity and speed as the rest of the symptoms during the prodromal and initial phases of schizophrenia. Recently, there has been a renewed interest in depression as an adaptive response to the changes that happen after a psychotic episode and its impact on the patient's social role, the stigma, and the feelings of shame and loss that overcome an individual in this situation $[9,10]$.

Nowadays, where psychosis and emotions seem to be divorced in the new classification of the fifth edition of the Diagnostic and Statistical Manual of Mental Disorders (DSM-5) [11], a door has been opened to a more dimensional perspective of psychosis and to numerous studies on productive and negative symptoms, social skills, insight, and very rigorous clinical trials to learn about the forms of the disorder and the processes of improvement through combined therapies; however, this has not translated into a special interest in PPD [12].

This pendular evolution in its pathogenesis tells us that it is a complex phenomenon itself, providing ways of explaining the characterization of the emotional problems that appear in psychosis as well. Therefore, difficulty in understanding how it is generated gives rise to doubts when considering PPD as an entity of its own, as part of a process or as a picture that can be presented as comorbid to psychosis $[13,14]$. 
Classification systems do not reach an agreement. The first reference to this disorder was found in the Diagnostic Research Criteria of Spitzer et al. [15].

As a consequence of this, PPD was first included in the tenth Classification of Mental and Behavioural Disorders (International Classification of Diseases [ICD]-10) in 1992 as F20.4 post-schizophrenic depression [16]. The definition requires that the patient meets the general criteria for schizophrenia during the previous 12 months and still exhibits the persistence of hallucinations, thought disorders, or negative symptoms that are not due to depression or neuroleptic medication. These criteria include many of the contents of the theoretical assumptions described in the introduction. Although it is understood that PPD occurs after the remission of an acute episode of psychosis, these depressive symptoms can occur before the positive symptoms of a psychotic episode, and according to some authors, in the current systems, they are not endowed with specificity or predictive value [17]. To distinguish PPD from prominent negative symptoms such as apathy, anhedonia, and sociality, criteria A1 of major depression (depressed mood) and simply A2 (loss of interest) are used.

Considering PPD is not part of the usual nomenclature of DSM-IV, people affected by this syndrome should be diagnosed with schizophrenia and unspecified depressive disorder (WHO [16]). It was not until 1994 when the DSM-IV and DSM-IV-R classifications were assigned the diagnosis of "unspecified depressive disorder"; however, despite not accepting the term "post-psychotic depression" as part of their nosological classification, they include it in their Appendix B as a research proposal. Despite this, the term has not been included in the recently published DSM-5, and little has been published about the aforementioned research proposed in the DSM-IV [18].

After the ICD defined the concept of post-schizophrenic depression, only a few more revisions were published on the term. An advance that made a difference in the way of studying and measuring PPD was the introduction of the Calgary Depression Scale for Schizophrenia (CDSS), specifically designed for subjects with PPD $[18,19]$.

In this line, it is essential to highlight the Birchwood's studies, to which we will refer in this review, which provide the following definition of PPD: PPD occurs following the remission of acute psychosis, where the individual (a) shows "at least moderate depression" (Beck Depression Inventory [BDI] greater than or equal to 15), (b) does not show a concomitant increase in psychotic symptoms, and $(c)$ is preceded by a sub-threshold (non-depressed) phase [20].
The main objective of this research was to review the published literature on PPD. The second objective was to indicate its clinical importance and prevalence rate, either comorbid or as an entity of its own. In order of answering these questions, we made a historical review of the term and a valid definition of PPD.

\section{Methods}

The recommendations of the Preferred Reporting Items for Systematic Reviews and Meta-Analyses (PRISMA): The PRISMA statement [21] was followed to achieve the aims of this review. Ethical approval was not required because this was a systematic review of published studies. This review was prospectively registered on PROSPERO (number CRD42020185534).

\section{Selection Criteria of the Articles}

The inclusion criteria of the studies were: (1) quantitative or qualitative research, review articles, and meta-analyses that examined the term PPD, (2) English and Spanish language articles, and (3) patients of all ages. The exclusion criteria were: (1) letters to the editor, opinions or commentaries, case reports, and short communications; (2) studies that included patients with other diagnoses in addition to PPD and that did not separate the results according to the diagnosis; and (3) articles focused only on the treatment of PPD.

\section{Search Strategy}

The PubMed database was searched until 1 November 2019. The search strategy used in these databases was the following MeSH terms: "post psychotic" AND "depr*” OR "post" AND "psych*” AND "psich" AND "depr." Filters were used in the database to meet the inclusion criteria.

\section{Study Selection Process}

This process was conducted in 4 phases. Firstly, in the identification phase, duplicate articles were removed. Secondly, in the screening phase, 2 reviewers (M.G.-J. and L.G.-R.) independently read the titles and abstracts of the articles that potentially met the inclusion criteria. A reasoned discussion with one another solved any disagreements; when there was no agreement, the full text of the doubtful article was reviewed. Thirdly, in the eligibility phase, the same reviewers examined and independently read the full-text articles that were preselected in the previous phase and the doubtful articles (including the reference lists of all these studies). Any disagreements were resolved through a reasoned discussion. Finally, in the inclusion phase, the reviewers selected the articles for inclusion in this systematic review.

\section{Data Extraction Process}

Two reviewers (M.G.-J. and L.G.-R.) independently analysed all of the selected articles, and the following information was extracted: (1) title of the study, author(s), and year of publication; (2) research design; (3) journal; (4) definition of PPD; and (5) key findings of patients with PPD. Some articles that did not appear in the review were added as multiple references were made to them in the reviewed articles due to their relevance. 
Fig. 1. Article selection process.

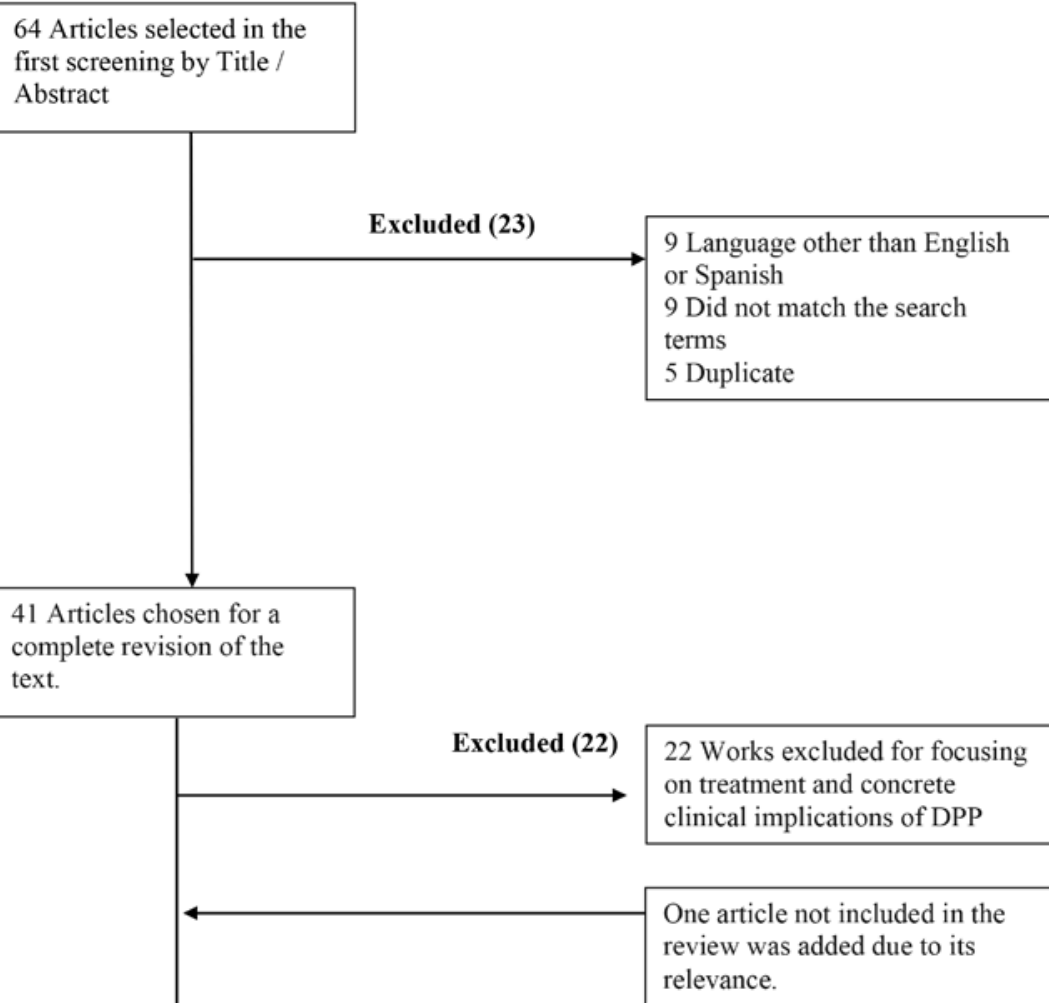

20 Works finally included in

the review

\section{Results}

The search in PubMed resulted in 64 articles, of which 9 were screened out based on the title. Four articles were removed after screening for duplicates. Nine of the 51 articles were eliminated because they were written in French, Russian, and Romanian, and 5 of them were duplicated.

Forty-one articles were finally selected for a complete review of the text. Twenty-two works were excluded because they were only focused on treatment or specific implications of PPD, such as suicide risk. Nineteen studies that met the inclusion criteria were finally selected [2, 20, 22-38]: Das and Kapur [22], Berrios and Bulbena [23], Becker [24], Leff et al. [25], Siris et al. [26], Chintalapudi et al. [27], Birchwood et al. [20], Iqbal et al. [28], Jeczmien et al. [2], Candido and Romney [29], Kohler and Lallart [30], Iqbal et al. [31], Birchwood et al. [32], Schwartz-Stav et al. [33], Sandhu et al. [34], Upthegrove et al. [35], Potik [36], Kjelby et al. [37], and Moritz et al. [38].
One article that did not appear in the search was added due to its relevance, and this article was found in the literature review as it was mentioned in several articles (McGlashan and Carpenter [1]). Twenty articles were finally included in the review. Figure 1 shows the selection process of these articles. The main characteristics of the articles are shown in Table 2.

Different types of studies were selected; 5 of them were reviews $[1,2,24,30,36]$, while the rest were clinical studies with patients, of which 8 of them were observational prospective $[20,22,25,28,31,32,37,38], 5$ were case/ control [23, 26, 27, 29, 33], 1 was a cohort study [35], and 1 was a qualitative study [34]. The selected studies included a total of 724 patients, some of which were different studies conducted on the same samples. Three of them were made with first episode of psychosis (FEP) patients $[31,34,35]$.

The selected measures to diagnose patients with PPD were different among the studies. The main difference 


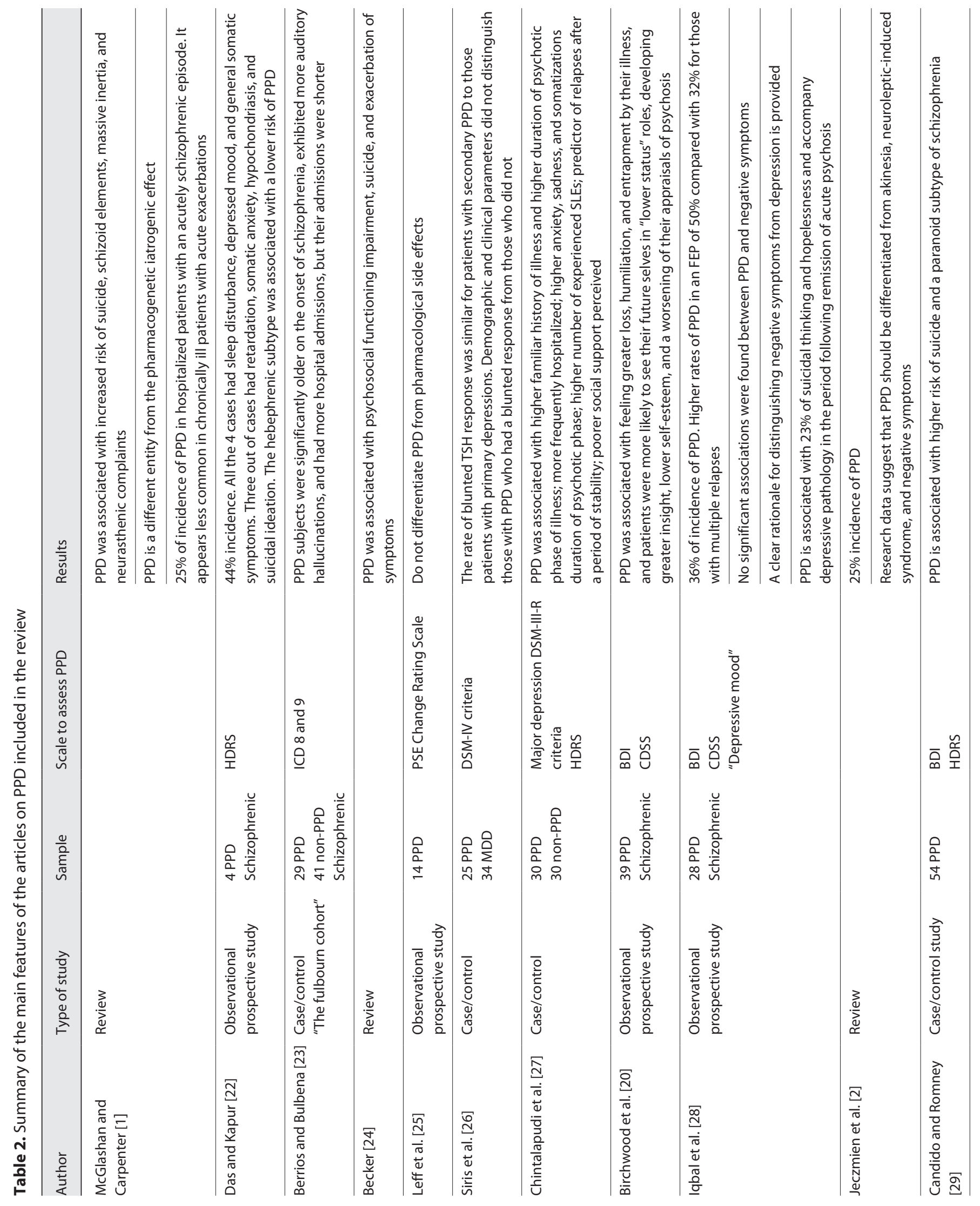




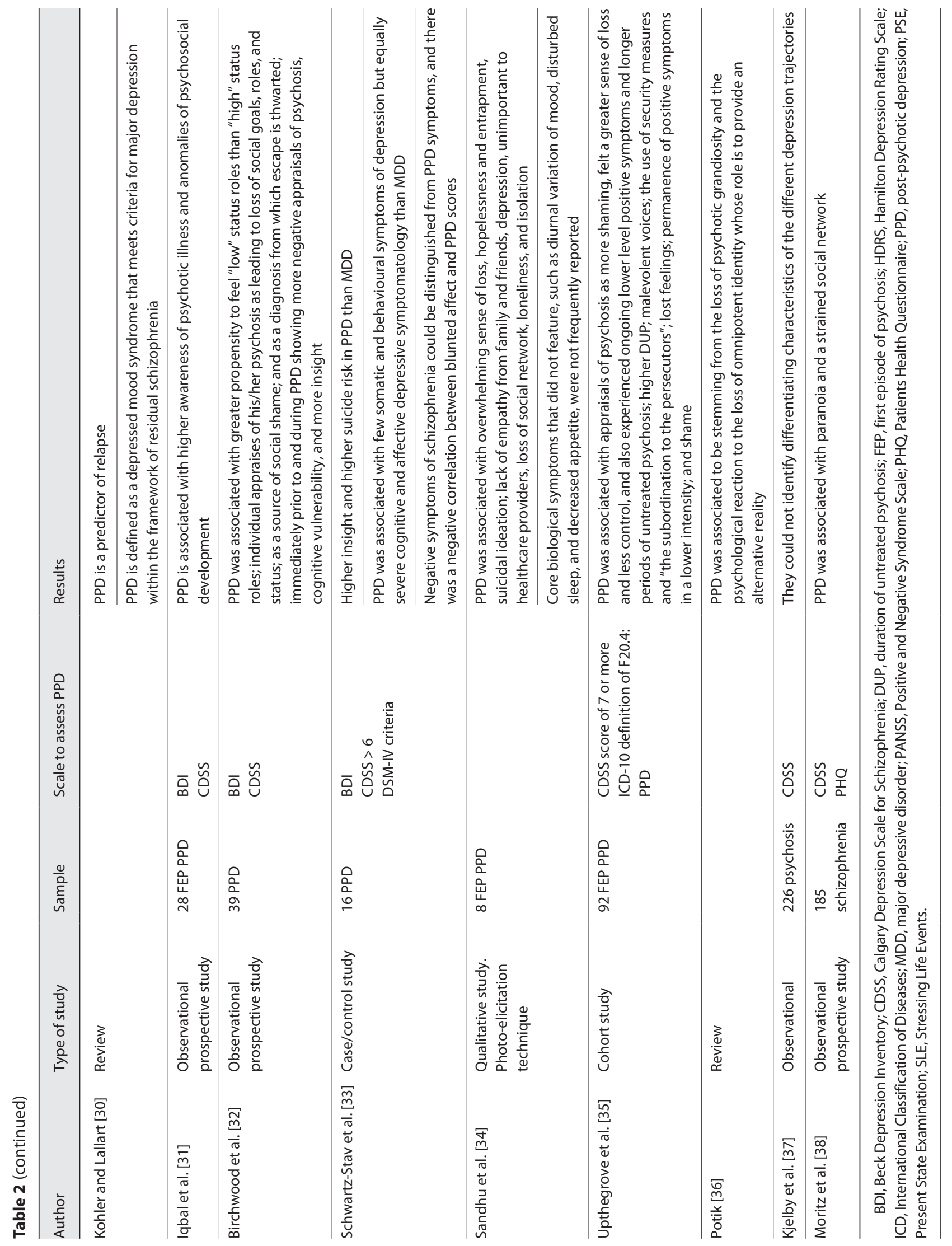


was that it was not until 2001 when research began to be carried out in which the CDSS was put to use, included thereafter in all subsequent studies $[20,28,31-33,35,37$, 38]. Most of the studies, but not all, diagnosed PPD when patients score more than 6-7 points on this scale. Studies pre-CDSS used the Hamilton Depression Rating Scale (HDRS), the BDI, and the DSM-IV criteria for PPD [39]. Only one study assessed the symptoms with the Present State Examination Change Rating Scale [40], while some studies used more than one of these scales.

\section{Epidemiology of PPD}

The incidences estimated in the selected articles showed estimations from a minimum of $25 \%[1,2]$ to $44 \%$ [22] with medium values of $36 \%$ [20]. Higher rates of incidence (50\%) were shown in patients with an FEP [28].

\section{Definition of PPD}

First of all, we found 3 types of depressive symptoms in psychoses:

- Firstly, the depressive symptoms defined as nuclear by Bleuler and that begin while the delusion is brewing [3]

- Secondly, depressive symptoms and major depression throughout the disorder in patients with psychosis

- Finally, the depressive symptoms that appear after the psychotic episode. We will elaborate its differential characteristics throughout the next revision, and we summarized later in a table (Table 2 ).

PPD is a different entity from the pharmacogenetic iatrogenic effect and the deterioration and negative symptomatology characteristic of this disorder $[1,2]$. The main difference with negative symptoms is that in addition to symptoms such as apathy or lack of interest, a depressive mood must be present.

PPD represents a syndrome of depressed mood, which meets the criteria for major depression, in the setting of residual schizophrenia. Heterogenic results are reported. The lack of tested diagnostic criteria has contributed to these findings and a misinterpretation of its relatively high frequency.

For this reason, the term "post-psychotic depression" has not been homogeneously used among investigations; however, since it was first defined, there has been a tendency to assume the Birchwood theory and definition of PPD.

PPD has been associated with some differential characteristics such as greater neurasthenic and schizoid components, as differential characteristics concerning other types of depression and less behavioural depressive symptoms but the same cognitive and affective symptoms to major depressive disorder (MDD). Regarding the somat- ic symptoms of depression, there are contradictory results $[27,33]$.

We found in this review an article that views PPD as a psychological response to the changes that occur during this stage in the person's life [37]. The author pointed out that many people diagnosed with schizophrenia feel that they are denied any hope of recovery and that they are marked with the stigma of mental illness, feel estranged, and suffer from depression, emptiness, and lack of meaning in life.

\section{Risk and Prognosis Factors of PPD}

The reported risk factors for presenting PPD were higher age [23], a higher familiar history of illness, a longer duration of untreated psychosis, and a higher number of experienced stressful life events [27]. Additionally, a higher incidence of PPD was reported in an FEP than in individuals who have experienced more than one relapse $[1,28]$. Moreover, a high insight at the beginning of psychosis increases the risk of the presentation of a PPD episode and suicide risk [31]. Social variables also play an important role, such as abnormalities in psychosocial development, lower status roles, social networks affected by psychosis, and, in general, lower social support that confers greater vulnerability to suffering from PPD [20,27].

The schizophrenia subtype confers different risks of PPD. The paranoid subtype is associated with an increased risk of developing PPD and increases the risk of suicide [29, 38]; auditory hallucinations and "malevolent voices," the use of security measures, the "subordination to persecutors," shame and sense of loss, and the schizoid component were associated as well $[1,23,35]$. However, the hebephrenic subtype, as well as a lower previous intellectual level, is associated with a lower incidence, so they act as protective factors to prevent PPD [22].

No differences in the $\mathrm{H}-\mathrm{H}-\mathrm{T}$ axis were found by studies with TSH and TRH stimulation in patients with PPD versus non-PPD [26]. Meanwhile, as prognosis factors, suicide risk was the most frequently reported association with PPD [1, 22, 28, 29, 31, 34], while a higher number of hospital admissions [27], the association with a higher chance of relapse [30], and worse quality of life [31] were reported to be associated with PPD.

\section{Discussion}

\section{Definition}

To start, to diagnose PPD, a differential diagnosis must be made. First of all, we underlined that the pathologies
88

Psychopathology 2022;55:82-92 DOI: $10.1159 / 000520985$
Guerrero-Jiménez et al. 
previously ruled out must be taken into account: bipolar disorder, schizoaffective disorder, and depressive episode with psychotic symptoms. It is important to point out that some of the studies in this review demonstrated that PPD is a different entity from the pharmacogenetic iatrogenic effect (there are descriptions about the term before the use of antipsychotics) and from the deterioration and negative symptomatology characteristics of this disorder $[1,2]$.

Among the different psychiatric nosology classification systems, there are disagreements and a lack of consensus when defining PPD criteria. This is due to a lack of agreement between the authors investigating this syndrome. Therefore, there is not a consensus regarding the definition of the depressive symptomatology that appears after an acute episode of psychosis in a schizophrenic psychotic disorder. Nevertheless, the first studies on the term attempted to classify PPD as an entity other than comorbidity between the previous psychotic picture and the subsequent depressive symptoms. It seems that the subsequent tendency is to describe it as a depressive episode that appears just after the psychotic symptoms. However, this type of depression seems to have different connotations to other types of depression such as major depression or that may occur at other times in the evolution of the psychotic disorder. As Becker and collaborators [24] pointed out, the lack of tested diagnostic criteria has led to a misinterpretation of its relatively high frequency and its association with characteristic poor results, such as deterioration of psychosocial functioning, exacerbation of symptomatology, and suicide. It affects the importance of differential diagnosis, partly because the evidence does not support the concept of schizophrenic depression as post-psychotic. Therefore, it seems relevant to classify it as a separate entity because of its clinical connotations, such as poorer outcome, suicide risk, and being a relapse predictor [30] although the use of the term has tended to be abandoned in recent decades.

The authors of this review described a series of differential symptoms that would be more characteristic of PPD than of other types of depression, such as a greater neurasthenic and schizoid component as differential characteristics concerning other types of depression and less behavioural depressive symptoms but the same cognitive and affective symptoms to MDD. Our results show that PPD would be a type of depression that would have greater neurasthenic and schizoid components and less behavioural depressive symptoms than MDD (such as agitation, given that it refers to behavioural symptoms of depression as measured by the BDI [39]). However, the main difference would be the prognostic difference conferred by whether it arises from the previous psychotic episode, which is mainly related to preoccupation and awareness of what happened and whether they continue to have latent psychotic symptoms. Regarding the somatic symptoms of depression, there were contradictory results $[27,33]$. However, these differential characteristics are associated, in our opinion; they do not seem to have sufficient importance by themselves to serve as a clinical diagnosis to differentiate them from other types of depression.

In this line of thought, certain premorbid factors could predispose to presenting PPD, as well as the psychological consequences that suffering a psychotic episode would have for the patients. Therefore, the authors conclude that the definition provided by Birchwood seems the most consistent with these findings [20, 28, 32]. According to the Birchwood definition, the diagnostic criteria obey the temporary criteria related to psychosis rather than specific characteristics of the depressive episode itself, which should have at least a moderate intensity: PPD occurs following the remission of acute psychosis, where the individual (a) shows "at least moderate depression" (BDI greater than or equal to 15), (b) does not show a concomitant increase in psychotic symptoms, and (c) is preceded by a sub-threshold (non-depressed) phase [20].

Then, a psychological model of PPD can be suggested, which includes a cognitive process of regained insight and an appraisal of illness, as well as the impact of diagnosis as a label itself. This conception of the PPD is at odds with the ICD-10 definition of PPD because although PPD is embedded in the realities of a psychotic illness, it is the beliefs or appraisals about psychosis made by those who experience it that are important $[20,28,41]$.

Psychoanalytical explanations are in line with this theory, suggesting possible implications for schizophrenic patients at different stages of the recovery process [36]. It has been pointed out that many people diagnosed with schizophrenia feel that they are denied any hope of recovery [42] and that they are marked with the stigma of mental illness, feel estranged, and suffer from depression, emptiness, and lack of meaning in life. Again, disease awareness appears or, as other psychoanalysts have previously called it, "the narcissistic wound" causes psychosis [6]. A person with schizophrenia can embody Kohut's belief in the "tragic man" more than in other conditions [13]. Potik pointed out that certain models of attention, such as common observation and empathic understanding, can help in the treatment [36]. As other psychoanalytic authors had previously postulated, delusions of 
grandeur or hallucinations immediately follow a wound to the patient's self-esteem [43]. The great content of thought or perception is the effort of the patient to counteract the narcissistic wound, and "when the delirium disappears, the patient becomes depressed."

\section{Risk and Prognostic Factors of PPD}

Clinical sample studies have demonstrated that even when psychotic symptoms occur isolated, they are associated with increased depression risk [22]. Phenomenological studies of auditory hallucinations have revealed that between two-thirds and three-quarters of people with auditory hallucinations are at least moderately depressed and distressed by psychotic experience. We hypothesize that these data would indicate that psychotic symptoms, across their gradient of intensity and variability, may be a risk factor for depressive symptoms also of varying intensity, which is supported by Birchwood and Freeman's theories [44-46].

The schizophrenia subtype seems to confer different risks of PPD; in line with the psychoanalytic theory of vulnerability that could confer delirium to depression, the paranoid subtype has been linked with an increased risk of developing PPD and increases the risk of suicide [22, 28, 29, 34]; auditory hallucinations and "malevolent voices," the use of security measures, the "subordination to persecutors," shame, and a sense of loss are associated as well $[23,35]$. However, the hebephrenic subtype, as well as a lower previous intellectual level, is associated with a lower incidence, so they act as protective factors to prevent PPD [22].

Several authors agree that paranoia probably interferes with social and work environments and that any of these challenges can lead to depressive symptoms through feelings of shame, entrapment, and a reduction of perceived social status [46]. Social variables play an important role in the development of PPD, this is congruent with other studies that have reported that abnormalities in psychosocial development could confer greater vulnerability to suffering from PPD [47]; worsening in prognosis, a worse quality of life, an increased risk of suicide [31] and a greater number of hospital admissions have been associated too [48], as was already happening in the Chintalapudi et al. [27] review. Loneliness and social isolation, feelings of loss and shame, and maintenance of positive symptoms, although at a low level and for a longer duration of untreated psychosis, are associated with the presentation of PPD $[34,35]$. Factors such as being Black despite having a higher incidence of an FEP and worse clinical results, having a lower feeling of loss and shame after an FEP, and therefore having a lower risk of PPD point to a possible relationship with alternative models of beliefs in health and strong relationships between patients and caregivers [49].

Given these associated factors that would aggravate the prognosis, patients, especially those experiencing an FEP, should be monitored for PPD/hopelessness/suicidal risk a few weeks after the acute episode. Patients' aspirations and perceived losses should be assessed and undergo therapy. Therapeutic interventions aimed at dealing with illness awareness and stigma through individual and family psychoeducation interventions $[50,51]$ as well as therapeutic approaches focussing on the recovery model based on its principles (giving hope, facilitating self-management, and helping the patient to have a positive identity and to take responsibility and control of their illness) $[51,52]$ seem to be key in the management of PPD and its prevention.

In most recent studies, Birchwood's definition of PPD has been set as the basis to investigate this type of depression, especially in an FEP, showing important clinical implications that are crucial in the recuperation process of an FEP. We think that a dimensional rather than categorical point of view must be adopted to understand the term PPD, taking into account an important dimension of depressive symptoms [53], in addition to the familiar dimensions of positive and negative symptoms.

Despite its high prevalence, the term has not been included in recent classification manuals and is tending to be abandoned. The data suggest that PPD is a nosological entity different from a secondary effect to antipsychotics, the negative symptoms of psychosis, and other clinical disorders that combine psychotic and depressive symptoms such as bipolar disorder, schizoaffective disorder, or depression with psychotic symptoms.

This review adds evidence to the theory that affective and psychotic symptoms are more closely associated than assumed from a "Kraepelinian" viewpoint. PPD represents a syndrome of depressed mood, which meets the criteria for major depression, in the setting of residual schizophrenia. The lack of tested diagnostic criteria has led to a misinterpretation of its relatively high frequency, as well as its association with poorer outcomes such as psychosocial functioning aggravation, symptomatology exacerbation, and higher suicide risk. It affects the importance of differential diagnosis, partly because the evidence does not support the concept of schizophrenic depression as post-psychotic. It seems relevant to classify it as a separate entity because of its clinical connotations such as poorer outcome, suicide risk, and a relapse pre-
Guerrero-Jiménez et al. 
dictor. Family history of illness, longer duration of untreated psychosis, FEP, a higher number of experienced stressing life events, higher insight at the beginning of psychosis, social abnormalities in psychosocial development, low-status roles, social network affected, and in general lower social support would confer greater vulnerability to suffering from PPD.

Psychotic symptoms, especially paranoid symptoms, probably interfere with the social and work environment and any of these challenges can lead to depressive symptoms through feelings of shame, entrapment, and a reduction of perceived social status and support. Therefore, psychosis treatments should aim to improve emotional well-being, including depression, whether or not they are related to future positive symptoms such as paranoia. A better understanding of PPD depression, especially in FEPs, could lead to better-targeted therapies and improved patient outcomes.

\section{Acknowledgments}

The authors would like to gratefully thank the collaboration of Department of Psychiatry members in the University of Granada.

\section{Statement of Ethics}

The authors declared that for this research, they did not carry out studies including human participants. This review article does not need a consent form to participate. The international recom- mendations were followed according to the Preferred Items for Reporting of Systematic Reviews and Meta-Analyses (PRISMA). This review was prospectively registered on PROSPERO (number CRD42020185534).

\section{Conflict of Interest Statement}

The authors declare that there is no conflict of interest regarding the publication of this article.

\section{Funding Sources}

This research did not receive any specific grant from funding agencies in the public, commercial, or not-for-profit sectors.

\section{Author Contributions}

All the authors had participated in the design of the study, collected the data, participated in the interpretation of the data, and the drafting of the article; they approved the final version of the manuscript.

\section{Data Availability Statement}

This research is a review article. All data generated or analysed during this study are included in this article. Further enquiries can be directed to the corresponding author.

\section{References}

1 McGlashan TH, Carpenter WT. Postpsychotic depression in schizophrenia. Arch Gen Psychiatry. 1976;33:231-9.

2 Jeczmien P, Levkovitz Y, Weizman A, Carmel Z. Post-psychotic depression in schizophrenia. Isr Med Assoc J. 2001;3:589-92.

3 Bleuler E. Dementia praecox or the group of schizophrenias. Translated by: J. Zinkin: New York, NY: International Universities Press; 1950.

4 Mayer-Gross W. Ueber die stellungnahme zur abgelaufenen akuten psychose. Eine studie über verständliche zusammenhänge in der schizophrenie. Z Gesamte Neurol Psychiatr. 1920;60:160-212.

5 Eissler KR. Remarks on the psychoanalysis of schizophrenia. Int J Psychoanal. 1951;32: 139-56; passim.

6 Semrad EV. Long-term therapy of schizophrenia. In: Usdin GL, editor. Psychoneuroses and schizophrenia. Philadelphia: J. B. Lippincott; 1966. p. 155-73.
7 Mino Y, Ushijima S. Postpsychotic collapse in schizophrenia. Acta Psychiatr Scand. 1989;80: $368-74$.

8 Jaspers K. General psychopathology. Manchester: Manchester University Press; 1963.

9 Conrad K. Die beginnende schizophrenie. Versuch einer gestaltanalyse des wahnsinns [In incipient schizophrenia: an attempt at a gestaltic analysis of insanity]. Stuttgart: Thieme; 1966.

10 Harrison G, Hopper K, Craig T, Laska E, Siegel $\mathrm{C}$, Wanderling J, et al. Recovery from psychotic illness: a 15- and 25-year international followup study. Br J Psychiatry. 2001;178:506-17.

11 American Psychiatric Association. Diagnostic and Statistical Manual of Mental Disorders. 5th ed. Washington: American Psychiatric Association; 2013.

12 American Psychiatric Association. Diagnostic and statistical manual of mental disorders. 5th ed. Arlington: American Psychiatric Press; 2013.
13 Jackson $\mathrm{H}$. Using self psychology in psychotherapy. Oxford: Jason Aronson Incorporated; 1994.

14 Lewis M. Child and adolescent psychiatry: a comprehensive textbook. Philadelphia: Lippincott Williams \& Wilkins Publishers; 2002.

15 Spitzer RL, Endicott J, Robins E. Clinical criteria for psychiatric diagnosis and DSM-III. Am J Psychiatry. 1975;132:1187-92.

16 World Health Organization (WHO). The ICD-10 classification of mental and behavioural disorders. Geneva: World Health Organization; 1992.

17 Malla AK, Norman RM. Prodromal symptoms in schizophrenia. Br J Psychiatry. 1994; 164:487-93.

18 Addington D, Addington J, Schissel B. A depression rating scale for schizophrenics. Schizophr Res. 1990;3:247-51.

19 Addington D, Addington J, Maticka-Tyndale E, Joyce J. Reliability and validity of a depression rating scale for schizophrenics. Schizophr Res. 1992;6:201-8. 
20 Birchwood M, Iqbal Z, Chadwick P, Trower P. Cognitive approach to depression and suicidal thinking in psychosis. 1. Ontogeny of post-psychotic depression. Br J Psychiatry. 2000;177:516-21.

21 Moher D, Shamseer L, Clarke M, Ghersi D, Liberati A, Petticrew M, et al. Preferred reporting items for systematic review and metaanalysis protocols (PRISMA-P) 2015 statement. Syst Rev. 2015;4:1.

22 Das P, Kapur RL. Post-psychotic depression in schizophrenics: a prospective study. Indian J Psychiatry. 1980;22:277-82.

23 Berrios GE, Bulbena A. Post psychotic depression: the fulbourn cohort. Acta Psychiatr Scand. 1987;76:89-93.

24 Becker RE. Depression in schizophrenia. Hosp Community Psychiatry. 1988;39:126975.

25 Leff J, Tress K, Edwards B. The clinical course of depressive symptoms in schizophrenia. Schizophr Res. 1988 Jan-Feb;1(1):25-30.

26 Siris SG, Frechen K, Strahan A, Cutler J, Owen K, Alvir J, et al. Thyroid releasing hormone test in schizophrenic patients with post-psychotic depression. Prog Neuropsychopharmacol Biol Psychiatry. 1991;15:36978.

27 Chintalapudi M, Kulhara P, Avasthi A. Postpsychotic depression in schizophrenia. Eur Arch Psychiatry Clin Neurosci. 1993;243. 103-8.

28 Iqbal Z, Birchwood M, Chadwick P, Trower P. Cognitive approach to depression and suicidal thinking in psychosis. 2. Testing the validity of a social ranking model. Br J Psychiatry. 2000;177:522-8.

29 Candido CL, Romney DM. Depression in paranoid and nonparanoid schizophrenic patients compared with major depressive disorder. J Affect Disord. 2002;70:261-71.

30 Kohler CG, Lallart EA. Postpsychotic depression in schizophrenia patients. Curr Psychiatry Rep. 2002;4:273-8.

31 Iqbal Z, Birchwood M, Hemsley D, Jackson C, Morris E. Autobiographical memory and post-psychotic depression in first episode psychosis. Br J Clin Psychol. 2004;43:97-104.
32 Birchwood M, Iqbal Z, Upthegrove R. Psychological pathways to depression in schizophrenia: studies in acute psychosis, post psychotic depression and auditory hallucinations. Eur Arch Psychiatry Clin Neurosci. 2005;255:202-12.

33 Schwartz-Stav O, Apter A, Zalsman G. Depression, suicidal behavior and insight in adolescents with schizophrenia. Eur Child Adolesc Psychiatry. 2006;15:352-9.

34 Sandhu A, Ives J, Birchwood M, Upthegrove $\mathrm{R}$. The subjective experience and phenomenology of depression following first episode psychosis: a qualitative study using photoelicitation. J Affect Disord. 2013;149:166-74

35 Upthegrove R, Atulomah O, Brunet K, Chawla R. Cultural and social influences of negative illness appraisals in first-episode psychosis. Early Interv Psychiatry. 2013;7:399-406.

36 Potik D. Self psychology conceptualization of postpsychotic depression and recovery among paranoid schizophrenic patients. Bull Menninger Clin. 2014;78:70-86.

37 Kjelby E, Gjestad R, Sinkeviciute I, Kroken RA, Løberg EM, Jørgensen HA, et al. Trajectories of depressive symptoms in the acute phase of psychosis: implications for treatment. J Psychiatr Res. 2018;103:219-28.

38 Moritz S, Schmidt SJ, Lüdtke T, Braunschneider LE, Manske A, Schneider BC, et al. Postpsychotic depression: paranoia and the damage done. Schizophr Res. 2019;211:79-85.

39 American Psychiatric Association. Diagnostic and statistical manual of mental disorders. 4th ed. Washington: American Psychiatric Press; 1994.

40 Tress KH, Bellenis C, Brownlow JM, Livingston G, Leff JP. The present state examination change rating scale. Br J Psychiatry. 1987;150: 201-7.

41 Birchwood M, Iqbal Z. Depression and suicidal thinking in psychosis: a cognitive approach. In: Wykes T, Tarrier N, Lewis S, editors. Outcome and innovation in psychological management of schizophrenia. Chichester: John Wiley and Sons Ltd; 1998. p. 56-76.
42 Deegan PE. Recovery and empowerment for people with psychiatric disabilities. Soc Work Health Care. 1997;25:11-24.

43 Steinman I, Garfield D. Self psychology and psychosis: the development of the self during intensive psychotherapy of schizophrenia and other psychoses. London: Routledge; 2018.

44 Chadwick P, Birchwood M. The omnipotence of voices. A cognitive approach to auditory hallucinations. Br J Psychiatry. 1994;164:190201

45 Trower P, Birchwood M, Meaden A, Byrne S, Nelson A, Ross K. Cognitive therapy for command hallucinations: randomised controlled trial. Br J Psychiatry. 2004;184:312-20.

46 Freeman D, Startup H, Dunn G, Wingham G, Černis E, Evans N, et al. Persecutory delusions and psychological well-being. Soc Psychiatry Psychiatr Epidemiol. 2014;49:1045-50.

47 Kay SR, Lindenmayer JP. Outcome predictors in acute schizophrenia: prospective significance of background and clinical dimensions. J Nerv Ment Dis. 1987;175:152-60.

48 Addington J, Leriger E, Addington D. Symptom outcome 1 year after admission to an early psychosis program. Can J Psychiatry. 2003; 48:204-7.

49 Turner MH, Bernard M, Birchwood M, Jackson $\mathrm{C}$, Jones $\mathrm{C}$. The contribution of shame to post-psychotic trauma. Br J Clin Psychol. 2013;52:162-82.

50 Lysaker PH, Whitney KA, Davis LW. Awareness of illness in schizophrenia: associations with multiple assessments of executive function. J Neuropsychiatry Clin Neurosci. 2006; 18:516-20.

51 Brent BK, Giuliano AJ, Zimmet SV, Keshavan MS, Seidman LJ. Insight into illness in patients and caregivers during early psychosis: a pilot study. Schizophr Res. 2011;127:100-6.

52 Shepherd G, Boardman J, Slade M. Making recovery a reality. London: Sainsbury Centre for Mental Health; 2008

53 van Os J, Verdoux H, Maurice-Tison S, Gay B, Liraud F, Salamon R, et al. Self-reported psychosis-like symptoms and the continuum of psychosis. Soc Psychiatry Psychiatr Epidemiol. 1999;34:459-63. 